\title{
A longitudinal study of cognitive predictors of (complex) post-traumatic stress in young people in out-of-home care
}

\author{
Rachel M. Hiller, ${ }^{1}$ (D) Richard Meiser-Stedman, ${ }^{2}$ (D) Elizabeth Elliott, ${ }^{1}$ Rosie Banting, ${ }^{1}$ and \\ Sarah L. Halligan ${ }^{1,3}$ (D) \\ ${ }^{1}$ Department of Psychology, University of Bath, Bath, UK; ${ }^{2}$ Department of Clinical Psychology, Norwich Medical \\ School, University of East Anglia, Norwich, UK; ${ }^{3}$ Department of Psychiatry and Mental Health, University of Cape \\ Town, Cape Town, South Africa
}

\begin{abstract}
Background: Young people in out-of-home care are substantially more likely to meet criteria for PTSD than their peers, while their early maltreatment exposure may also place them at greater risk of developing the newly proposed complex PTSD. Yet, there remains limited empirical evidence for the mechanisms that might drive either PTSD or complex features in this group, and ongoing debate about the suitability of existing cognitive behavioural models and their related NICE-recommended treatments. In a prospective study of young people in out-of-home care, we sought to identify demographic and cognitive processes that may contribute to the maintenance of both PTSD symptom and complex features. Methods: We assessed 120 10- to 18-year-olds in out-of-home care and their primary carer at two assessments: an initial assessment and 12-month follow-up. Participants completed questionnaires on trauma history, PTSD symptoms and complex features, while young people only also self-reported on trauma-related (a) maladaptive appraisals, (b) memory quality and (c) coping. Social workers reported on maltreatment severity. Results: Young people's maltreatment severity was not a robust predictor of either PTSD symptoms or complex features. All three cognitive processes were moderately-to-strongly correlated with baseline and 12-month PTSD symptoms and complex features, with maladaptive appraisals the most robust unique driver of both, even when controlling for initial PTSD symptom severity. Conclusions: Existing cognitive models of PTSD are applicable in this more complex sample of young people. The model was also found to be applicable to the additional features of complex PTSD, with the same processes driving both outcomes at both time points. Clinical implications are discussed. Keywords: Child maltreatment; foster care; complex trauma; developmental trauma; looked-after children; PTSD; complex PTSD.
\end{abstract}

\section{Introduction}

Most young people who have been removed from their family home and placed in out-of-home care have a history of maltreatment and trauma, including physical abuse, sexual abuse, emotional abuse, exposure to domestic violence and/or neglect (Department for Education, 2018). Typically, these experiences have occurred within a context of multiple other adversities, such as poverty, parental mental health difficulties, and parental drug and alcohol use (e.g. Oswald et al., 2009; Smith et al., 2007). Child maltreatment and adversity are key predictors of psychological difficulties across the life span (Keyes et al., 2012). While young people in care can be incredibly resilient in the face of significant challenges, there is well-documented evidence of substantial mental health difficulties in this group (e.g. Ford et al., 2007; Hiller \& St Clair, 2018). This includes that young people in care in the UK are five times more likely to meet criteria for any psychiatric disorder compared to their peers (Ford et al., 2007). While such outcomes are well-documented, and there is a growing body of qualitative evidence on the psychological needs of this group (e.g. see
Oswald et al., 2009; Rock et al., 2013), there has remained little quantitative evidence on mechanisms that may promote or hinder the psychological adjustment of young people in care. Such evidence is essential for informing targets for psychological support, particularly given the lack of consensus around the best treatment approaches for these young people.

The experience of maltreatment or trauma can lead to a range of potential mental health difficulties. One such outcome is post-traumatic stress disorder (PTSD), a trauma-specific mental health outcome (American Psychiatric Association, 2013). In DSM-5, PTSD is categorised by four core symptom clusters: intrusive symptoms (e.g. nightmares or flashbacks), avoidance (e.g. avoiding trauma-related thoughts or feelings), negative alteration in cognition and mood (e.g. exaggerated self-blame, negative affect) and alterations in arousal (e.g. irritability, hypervigilance; APA, 2013). Rates of PTSD in young people in care are estimated to be twelve times higher than in their peers (Ford et al., 2007). In response to the complex psychological presentation that can present alongside PTSD, particularly following ongoing or repeated trauma exposures, such as maltreatment, the World Health Organization's diagnostic classification system (ICD-11; WHO, 2018) has introduced 
a diagnostic category of complex PTSD (Brewin et al., 2017; Cloitre et al., 2013). Of note, this classification has not been introduced in DSM (APA, 2013). To meet criteria for complex PTSD, a young person must still meet full criteria for PTSD. Under ICD-11, this reflects three symptom clusters: re-experiencing, avoidance and hyper-arousal, and does not include the subscale 'alterations in cognition and mood', as found in DSM-5. The complex features of complex PTSD then comprise three additional symptom clusters: (a) affect dysregulation, (b) negative self-concept and (c) disturbances in relationships (WHO, 2018). While the infancy of complex PTSD means there is less evidence to support its applicability to young people in care, there is growing evidence that it forms a distinct diagnostic category from 'standard' PTSD, which is commonly related to child maltreatment (e.g. Karatzias et al., 2017; Sachser, Keller, \& Goldbeck, 2017).

Elevated rates of these trauma-specific mental health outcomes might be expected in young people in care, given exposure to interpersonal trauma is commonplace for this group, and such trauma exposure has been identified as the key predictor of whether or not a young person develops PTSD (Lewis et al., 2019). Potentially elevated rates of complex PTSD may also be expected, given the high prevalence of complex comorbidities that can present in these young people (Ford et al., 2007), and the fact that the three complex features are likely to be transdiagnostic symptoms (e.g. negative self-concept overlaps with depression; interpersonal difficulties are commonly found in other mental health disorders). If left unaddressed trauma-related mental health difficulties can become chronic, with substantial negative implications for broader well-being and development, including increased risk of developing other psychological difficulties and consequences for educational outcomes and relationships (Lewis et al., 2019) - all outcomes where young people in care often experience problems (DfE, 2018).

Cognitive and behavioural theories highlight several key psychological processes that may lead to, or maintain, PTSD symptoms (e.g. Brewin, Dalgleish, \& Joseph, 1996; Ehlers \& Clark, 2000; Foa, Steketee, $\&$ Rothbaum, 1989). The most widely cited of these models, the cognitive model of PTSD (Ehlers \& Clark, 2000), particularly focuses on three core psychological processes that may maintain a person's ongoing sense of threat, and ultimately PTSD symptoms. First, the individual forms negative appraisals about their trauma, which may generalise (e.g. I'll never get over what happened; I can't trust anyone; MeiserStedman et al., 2019). Second, how the memory was encoded and the resultant memory qualities are said to further maintain an ongoing sense of threat. For example, trauma memories are often encoded in such a way that they have more limited semantic, temporal or contextual information, but include many original emotions and sensory impressions (see Brewin, 2016). The resultant disorganised or fragmented trauma memories, along with sensoryheavy flashback style memories, can create a feeling of ongoing threat or re-experiencing (sometimes referred to as a feeling of 'nowness'). Third, engaging in maladaptive coping strategies, such as cognitive avoidance or thought suppression, is hypothesised to further exacerbate the ongoing sense of threat. There is well-established evidence for the role of these processes in maintaining PTSD in adult samples (e.g. Dunmore et al., 2001; Pietrzak, HarpazRotem, \& Southwick, 2011) and in samples of young people exposed to single-incident traumas (e.g. Bryant et al., 2007; Dalgleish et al., 2005; Hiller et al., 2018; Meiser-Stedman et al., 2019; Stallard \& Smith, 2007). Relatedly, psychological treatments that target these processes (e.g. trauma-focused cognitive behavioural therapy; Cohen et al., 2000) are the first-line recommended treatment for PTSD, with a good evidence base for adults, as well as children and young people (see NICE, 2018).

Despite substantial progress in understanding the role of these core processes in maintaining PTSD symptoms, there has been less attention to their relevance for more complex child trauma samples, where interpersonal trauma exposure has often been repeated and ongoing from a young age, and within the context of other adversity (Wiseman et al., 2019). Further, while there remains a need to establish stronger evidence for the relevance of cognitive models in relation to 'standard' PTSD symptoms, the introduction of complex PTSD also raises questions around what processes may maintain complex features and whether different treatment targets may be necessary. After ongoing multiple trauma exposures or maltreatment, it may be expected that maladaptive appraisals (e.g. The world is not safe) would be particularly entrenched. However, the potential role of memory qualities and coping remains unclear. In the weeks after single-incident trauma exposure, there is evidence that children's higher endorsement of disorganised and sensoryheavy trauma memories predict later PTSD symptom severity (e.g. Hiller et al., 2018; McKinnon et al., 2017). The picture may be less clear for young people in care, given, for example, many would experience disorganised trauma memories by virtue of the early developmental period within which some of the exposure is likely to have occurred. Further, while avoidant coping (e.g. thought suppression) is considered a key risk factor for PTSD (Trickey, Siddaway, Meiser-Stedman, Serpell, \& Field, 2012), there is some evidence that in contexts of ongoing risk or instability, potentially 'maladaptive' coping strategies such as avoidance, may actually be protective, at least in the shorter term (Gonzales et al., 2001; Grant et al., 2000). With a lack of empirical evidence on mechanisms that promote or hinder psychological adjustment of young people in out-ofhome care, there remains ongoing debate about the 
relevance of existing cognitive models for these more complex child trauma cases, and thus, their associated treatments (e.g. DeJong, 2010; Van der Kolk, 2017). Such concerns can have a substantial influence on decision-making around the best available treatment approaches for these young people and there remains an urgent need to develop evidence for the relevance of these models in complex groups to guide social care and mental health professionals in decision-making around assessments and therapeutic support (Lewis et al., 2019).

To address this, we ran a longitudinal study with young people in out-of-home care in the UK. Specifically, we explored the role of (a) maladaptive appraisals, (b) trauma memory qualities and (c) cognitive coping strategies, in relation to both young person and carer report of children's PTSD symptoms and complex features. The two key aims for the study were to establish whether existing cognitive models of PTSD would be applicable to young people in care, and whether the same processes that drive PTSD symptoms, also drive complex features.

\section{Methods}

\section{Participants}

We recruited 120 young people in care aged 10-18 years old and their primary carer, from three local authorities in England. In line with national statistics on young people in care (see DfE, 2018), the majority of participants (86\%) were in the care of a nonbiological foster carer, with $9 \%$ in kinship care (i.e. with a relative), and $5 \%$ in residential care homes, where their key worker provided carer report. The vast majority of young people were on full care orders. Demographics are presented in Table 1. Exclusion criteria were as follows: developmental difficulties that precluded mainstream schooling, significant current suicidal ideations and psychosis.

Table 1 Sample demographics

\begin{tabular}{|c|c|c|}
\hline & Young person & Carer \\
\hline Age in years, $M(S D)$ & $13.5(2.2)$ & $52.4(9.8)$ \\
\hline Female & $53 \%$ & $92 \%$ \\
\hline Caucasian & $83 \%$ & $94 \%$ \\
\hline $\begin{array}{l}\% \text { in long-term } \\
\text { placement }\end{array}$ & $89 \%$ & \\
\hline $\begin{array}{l}\text { Length of placement }{ }^{\mathrm{a}} \text {, } \\
M(S D), \text { Range }\end{array}$ & $\begin{array}{l}3.3(2.5) \\
1 \text { month - } \\
15 \text { years }\end{array}$ & - \\
\hline $\begin{array}{l}\text { \% young people not in } \\
\text { school }\end{array}$ & $10 \%$ & - \\
\hline Years as carer & - & $\begin{array}{l}7.0(7.6) \\
3 \text { months - } \\
35 \text { years }\end{array}$ \\
\hline \multicolumn{3}{|l|}{ Carer education level } \\
\hline $\begin{array}{l}\% \text { School until } \\
16 \text { yo or below }\end{array}$ & - & $28 \%$ \\
\hline $\begin{array}{l}\% \text { School until } \\
18 \text { yo }\end{array}$ & - & $10 \%$ \\
\hline$\%$ Higher education & - & $62 \%$ \\
\hline
\end{tabular}

${ }^{a}$ Length of time young person had been in placement at baseline (initial assessment).

\section{Procedure}

Ethical approval was obtained by the University of Bath and Social-Care Research Ethics Committees (Ref 16/IEC08/ 0025), as well as by participating Local Authorities. Potential participants were recruited via social workers, who have parental responsibility of the young person. In total, 242 young people were consented by their social worker. From this, 120 young person-carer pairs volunteered to participate (representing $49.5 \%$ of the originally social worker consented sample). There was no significant differences in terms of the age $(p=.21)$ or sex $(p=.73)$ of participants versus nonparticipants. Of the 122 nonparticipants, the most common reason for not participating was that the research team was unable to make contact in the study timeframe (39\%) and they were too busy $(38 \%)$. Participating young people provided informed assent (or consent if $16+$ years old), while carers provided informed consent for their own participation. For all assessments, the pair had the option of completing the questionnaire packs as a home visit or via post or online. Participants were invited to complete a 12-month follow-up, with $80 \%$ of the sample retained. There were no significant differences between those who did and did not participate in the 12-month followup in terms of sex $(p=.74)$, initial PTSD symptoms $(p=.82)$ or initial complex features symptoms $(p=.56)$, although there was a trend for age $(p=.059)$, with those who did not complete the follow-up being slightly older $(M=9.53, S D=3.62 \mathrm{v}$ $M=8.31, S D=3.51)$. A 6-month follow-up was also completed, but is not reported on here.

\section{Measures}

Trauma/maltreatment history. The young person and carer reported on the young person's trauma history on the 15item trauma history checklist of the Child and Adolescent Trauma Screen, where they ticked 'yes' or 'no' to various criterion A traumas (CATS; Sachser, Berliner et al., 2017). Social workers provided more detailed reporting on their maltreatment history using Kaufman's 5-point Likert scales, covering (a) physical abuse, (b) sexual abuse, (c) emotional abuse and (d) domestic violence (Kaufman et al., 1994). Here, scores ranged from 0 (young person did not experience that type of abuse) to 4 (most severe), with the exception of neglect which was rated by the social worker ticking a possible 10 types of neglect (score ranging from 0 to 10 ).

To describe the sample and allow for broad comparisons between young person, carer, and social worker report, their responses on the CATS (young person, carer) and Kaufman scale (social workers) were collapsed in to 'present' or 'absent' for (a) physical abuse, (b) sexual abuse and (c) domestic violence. The CATS does not include items on neglect or emotional abuse.

Post-traumatic stress symptoms. Following completion of the trauma history checklist, the young person and carer both reported on the young person's PTSD symptoms on the 20-item CATS DSM-5 PTSD symptom scale (Sachser, Berliner et al., 2017), with response options ranging from 0 (Never) to 3 (Almost Always). The measure produces both a total symptom scale and a 'probable PTSD' diagnosis based on endorsing 'Half the time' or 'Almost always' for the required number of symptoms corresponding to DSM-5. The measure showed strong internal consistency for both carer report and young person self-report $(\alpha>$.93). As part of our sensitivity analyses (described in detail later), we re-ran key complex features analyses with the 'altered mood and cognition' items removed from this checklist. This was because ICD-11 PTSD does not cover this subscale, as DSM-5 does. With these 8 items removed, the measure retained strong internal consistency $(\alpha=.90)$. 
Complex PTSD features. Young people and carers completed a 12-item Child Complex PTSD Checklist, a newly developed measure assessing three additional symptoms (beyond PTSD) of ICD-11 complex PTSD: negative self-concept interpersonal difficulties and affect dysregulation. Responses were rated on a 0 (Never) to 3 (Almost Always) scale. This scale was developed by experts in the child trauma field, based on adult scales and ICD criteria (WHO, 2018). The measure showed good internal consistency $(\alpha=.93)$. The measure is provided in Figure $\mathrm{S} 1$.

Maladaptive appraisals. Young people completed the self-report Child Posttraumatic Cognitions Inventory (CPTCI; Meiser-Stedman et al., 2009), a 25-item measure of maladaptive appraisals. Each item is rated on a 1 (Don't agree at all) to 4 (Agree a lot) scale $(\alpha=.96)$.

Coping. Young people completed the self-report Child Posttrauma Coping Questionnaire (CPCQ; Hiller et al., 2018), an 11 -item scale measuring rumination and cognitive avoidance. Each item is rated on a 0 (Not at all or only one time) to 3 (A lot of the time) scale $(\alpha=.91)$.

Trauma memory quality. Young people completed an adapted version of the Trauma Memory Quality Questionnaire (TMQQ; Meiser-Stedman et al., 2007). The adapted TMQQ (ATMQQ; Hiller et al., 2018) is an 18-item measure that includes the 11 original items from the TMQQ on the sensory quality of the trauma memory and sense of "nowness" and an additional 7 items on disorganised memories. Each item was rated on a 1 (Disagree a lot) to 4 (Agree a lot) scale $(\alpha=.88)$.

\section{Data analytic plan}

Data were analysed using SPSS. Our primary aims were to explore (1) whether the three cognitive processes (appraisals, memory, coping) would be associated with (a) concurrent and 12-month PTSD and (b) concurrent and 12-month complex features, and (2) whether these same processes would be associated with complex features, even after accounting for PTSD symptom severity. Both PTSD severity and complex features severity were positively skewed, so we applied a square root transformation. We then used preliminary bivariate and point biserial correlational analyses to understand the basic associations between the variables, as well as the role of age and sex as potential covariates. Variables were only included in the hierarchical linear regression models where correlations were identified $(p<.05)$. In all regressions, covariates were included in Step 1 and the cognitive processes (appraisals, memory, coping) were entered in Step 2. First, we explored the unique contribution of the baseline cognitive processes in relation to (1) concurrent PTSD symptoms and (2) 12-month PTSD symptoms. In particularly conservative analysis, we also explored whether cognitive processes would predict 12-month PTSD symptoms even after controlling for initial severity. Next, we explored whether these same cognitive processes explained variance in (1) concurrent complex features severity and (2) 12-month complex feature severity. In both cases, we also explored whether cognitive processes would still predict complex features, even when controlling for PTSD symptom severity.

To assess the robustness of the findings, we then ran three sensitivity analyses. First, we explored whether there were any discrepancies in findings following multiple imputation for missing data. The $20 \%$ missing data at 12 months were considered missing at random. Multiple imputation was performed using 50 iterations and predictive mean matching. Second, unlike the DSM-5, the ICD-11 PTSD diagnosis does not include an 'alterations in cognition and mood' symptom cluster. As complex PTSD is only defined in ICD-11, we re-ran the complex feature analyses with the 'altered mood and cognition' items removed from the PTSD symptom checklist, to explore whether moving between the two classification systems may have affected the results. As a third sensitivity analysis, we explored whether results were replicated when using carerreported symptom scores.

\section{Results Descriptives}

Young people (53\% female) were aged $10-18$ years old $(M=13.5$ years, $S D=2.2)$. On average, they had entered care at 8.3 years old $(S D=3.8$, Range birth 16 years old) and had been in care for between 6 months and 15 years $(M=4.9$ years, $S D=3.7)$. At the initial assessment, most participants were in a planned long-term placement $(89 \%)$. By the 12 month follow-up, $25 \%$ of the original sample were with a new carer. Carers at the original time point were aged 27-72 years old, were predominantly not biologically related to the young person $(91 \%)$ and were predominantly female (92\%). See Table 1 .

Maltreatment history. From self-, carer and social worker report, all young people had at least one source endorse maltreatment exposure. Seventyseven per cent of young people self-reported exposure to at least one criterion A trauma $(M=3.0$, $S D=2.9)$, with this increasing to $85 \%(M=3.4$, $S D=3.0$ ) from carer report. The proportion of maltreatment exposures endorsed by each reporting source is presented in Table 2. Using Cohen's Kappa analyses, there was poor agreement between child and social worker report $(\kappa=.07-.38)$, child and carer report $(\kappa=.16-.46)$, and social worker and carer report $(\kappa=.18-.47)$.

Symptom change, 'Probable' PTSD and agreement. The full correlation matrices are in Tables $\mathrm{S} 1$ and S2, where we also present the mean scores of

Table 2 Young person's maltreatment or trauma histories from their own, carer and social worker report

\begin{tabular}{|c|c|c|c|c|}
\hline & $\begin{array}{l}\text { Child } \\
\text { report }\end{array}$ & $\begin{array}{l}\text { Carer } \\
\text { report }\end{array}$ & $\begin{array}{l}\text { Social worker } \\
\text { report }\end{array}$ & Any $^{a}$ \\
\hline $\begin{array}{l}\text { Physical } \\
\text { abuse }\end{array}$ & $58 \%$ & $60 \%$ & $44 \%$ & $84 \%$ \\
\hline Sexual abuse & $15 \%$ & $25 \%$ & $18 \%$ & $32 \%$ \\
\hline $\begin{array}{l}\text { Domestic } \\
\text { violence }\end{array}$ & $50 \%$ & $72 \%$ & $77 \%$ & $94 \%$ \\
\hline $\begin{array}{l}\text { Emotional } \\
\text { abuse }\end{array}$ & - & - & $87 \%$ & - \\
\hline Neglect & - & - & $96 \%$ & - \\
\hline
\end{tabular}

Trauma history checklists did not assess for emotional abuse or neglect.

${ }^{a}$ Any reflects the proportion of the sample where any source (i.e. young person, carer or social worker) endorsed that the young person had experienced that type of maltreatment. 
measures at each time point. Based on paired samples $\mathrm{t}$ tests, there were no significant changes in self-reported PTSD symptoms from baseline $(M=13.6, S D=11.93)$ to 12 months $(M=12.81$, $S D=13.82, t=0.52, p=.60)$. Similarly, there was no significant change in scores on the complex feature measure from baseline $(M=8.42, S D=8.45)$ to 12 months $(M=7.76, \quad S D=8.68, \quad t=0.78$, $p=.44)$. From carer report, there was also no evidence of significant symptom change over the 1year period (PTSD symptoms - baseline: $M=16.80$, $S D=13.84 ; 12$ months: $M=14.04, S D=14.25$, $t=1.79, \quad p=.08 ;$ complex features - baseline: $M=11.48, \quad S D=9.18 ; \quad 12$ months: $M=10.31$, $S D=9.10, t=1.30, p=.20)$. Based on intra-class correlations with absolute agreement, there was generally poor agreement between child and carer report of total symptoms, at both time points for PTSD symptoms (baseline: $\mathrm{ICC}=.29 ; 12$ months: ICC $=46$ ) and complex features (baseline: ICC $=.42$, 12 months: $\mathrm{ICC}=.50)$. In all cases, mean scores showed carers tended to endorse a greater number of symptoms than the young person self-reported.

Based on DSM-5 criteria, at baseline $19 \%$ of young people met criteria for 'probable PTSD diagnosis' from self-report, while this increased to $25 \%$ from carer report. If based on either the carer or young person endorsing the symptom cluster (as is standard practice for diagnostic interviews), the rates of 'probable PTSD' increased to 32\%. Cohen's kappa analysis showed poor carer-child agreement for 'probable PTSD' $(\kappa=.38)$.

\section{Associations between variables}

Age, sex, PTSD symptoms and complex features. PTSD symptom severity and complex feature severity were strongly correlated with both time points $(r \mathrm{~s}>.74 ; p s<.001)$. When the DSM-only 'alterations in cognition and mood' subscale were removed from the PTSD symptom scale, PTSD symptom severity and complex feature severity remained strongly correlated $(r \mathrm{~s}>.70, p \mathrm{~s}<.001)$. Age of the child at baseline was not significantly associated with PTSD symptoms or complex features at either time point $(r s<.16, p s>.10)$. In contrast, the sex of the child was associated with PTSD symptoms and complex feature severity at both time points $\left(r \mathrm{~s}_{\mathrm{pb}}>.22, p \mathrm{~s}<.05\right.$; Table $\left.\mathrm{S} 1\right)$. In all cases, higher symptom severity was associated with being female. Sex was added as a covariate in all regressions.

Maltreatment history, PTSD symptoms and complex features. There was little evidence that the severity of the young person's maltreatment exposure (based on the continuous social worker scores for severity) was significantly associated with either PTSD symptoms or complex features at either time point $(r \mathrm{~s}<.15 ; \quad p \mathrm{~s}>.11$; Table $\mathrm{S} 1)$. The only exception to this was a significant association between sexual abuse severity and baseline PTSD symptom severity $(r=.26, p=.01)$. Similarly, there was no evidence that the age the young person entered care (as a proxy of length of maltreatment exposure) was significantly associated with either PTSD symptoms or complex features (Table S1).

Cognitive processes, PTSD symptoms and complex features. There were moderate to strong correlations between all three baseline cognitive processes (appraisals, memory, coping) and concurrent PTSD symptoms $(r \mathrm{~s}=.65-.77, p \mathrm{~s}<.001)$ and complex features $(r \mathrm{~s}=.50-.80, p \mathrm{~s}<.001)$, and 12 -month PTSD symptoms $(r \mathrm{~s}=.50-.56, \mathrm{ps}<.001)$ and complex features $(r \mathrm{~s}=.37-.52, p \mathrm{~s}<.001)$. Thus, all three processes were retained in the regression models (see Table S2).

\section{Linear regressions}

Concurrent PTSD symptoms. Cognitive processes explained a unique $59 \%$ of variance in concurrent PTSD symptoms, with maladaptive appraisals and cognitive coping (thought suppression, rumination), but not memory qualities, uniquely driving PTSD symptoms (Table 3).

12-month PTSD symptoms. Baseline cognitive processes explained a unique $29 \%$ of variance in 12 month PTSD symptoms, with maladaptive appraisals identified as the sole unique predictor $(\beta=.36$; Table 3). The model remained significant even when controlling for baseline PTSD symptoms, with cognitive processes continuing to explain a unique $7 \%$ of variance in 12 -month PTSD symptoms $(p=.04)$. No individual process was identified as a significant unique predictor, although there was a trend for maladaptive appraisals remaining the sole unique predictor $(\beta=.30, p=.06)$, while initial PTSD symptoms were not a unique predictor of 12 -month PTSD symptoms $(\beta=.12, p=.47)$.

Concurrent complex features. Cognitive processes explained a unique $62 \%$ of variance in complex feature severity. As with PTSD symptoms, maladaptive appraisals and coping, but not memory qualities, were significant unique predictors of concurrent complex features (Table 3).

After controlling for initial PTSD symptoms, the three cognitive processes continued to explain a significant $9 \%$ of variance in complex features. Here, initial PTSD symptoms and maladaptive appraisals were unique predictors of complex features, while memory quality and coping were not (Table 3).

12-month complex features. Initial cognitive processes continued to explain a unique $23 \%$ of variance in complex features, 12 months later. As with PTSD symptoms, maladaptive appraisals were the 
Table 3 Results of linear regressions for cognitive processes predicting child-reported baseline and 12-month PTSD symptoms and complex features

\begin{tabular}{|c|c|c|c|c|c|c|}
\hline & \multicolumn{3}{|c|}{$\begin{array}{l}\text { Model } 1 . \text { Controlling } \\
\text { for sex }\end{array}$} & \multicolumn{3}{|c|}{$\begin{array}{c}\text { Model } 2 \text {. Controlling } \\
\text { for baseline PTSD } \\
\text { symptoms }\end{array}$} \\
\hline & $R^{2} \Delta$ & $F \Delta$ & $\beta$ & $R^{2} \Delta$ & $F \Delta$ & $\beta$ \\
\hline \multicolumn{7}{|c|}{ Baseline PTSD symptoms } \\
\hline Appraisals & .59 & $58.15^{*}$ & $.47 *$ & & & \\
\hline Coping & & & $.27 *$ & & & \\
\hline Memory & & & $.15^{+}$ & & & \\
\hline \multicolumn{7}{|c|}{ 12-month PTSD symptoms } \\
\hline Appraisals & .29 & $12.53 *$ & $.36 *$ & .07 & $2.76 * *$ & $.30^{+}$ \\
\hline Coping & & & .16 & & & .14 \\
\hline Memory & & & .10 & & & .09 \\
\hline \multicolumn{7}{|l|}{ Baseline CF } \\
\hline Appraisals & .62 & $58.31^{*}$ & $.70 *$ & .09 & $9.30 *$ & $.45^{*}$ \\
\hline Coping & & & $.22 * *$ & & & $.16^{+}$ \\
\hline Memory & & & -.09 & & & $-.14^{+}$ \\
\hline \multicolumn{7}{|l|}{ 12-month CF } \\
\hline Appraisals & .23 & $7.90 *$ & $.41^{*}$ & .10 & $3.36 * *$ & $.45^{*}$ \\
\hline Coping & & & .08 & & & .10 \\
\hline Memory & & & .04 & & & .05 \\
\hline
\end{tabular}

Sex controlled for in Step 1 of each regression. Three cognitive processes entered in Step 2.

${ }^{*} p<.01 ; * * p<.05,{ }^{+} p<.10$.

sole unique cognitive predictor of later complex features (Table 3).

When controlling for baseline PTSD symptoms, cognitive processes continued to explain a unique $10 \%$ of variance in later complex features, with maladaptive appraisals remaining the sole unique predictor (Table 3). However, when controlling for initial complex feature severity, the cognitive processes no longer significantly predicted 12-month complex features $(p=.35)$, with initial complex features the only unique driver of later complex features $(\beta=.64, p=.003)$.

\section{Sensitivity analyses}

Multiple imputation. We used multiple imputation to account for the $20 \%$ missingness at 12 months (see Data Analytic Plan). The pattern of results was replicated (see Table S3, for details).

ICD-11 PTSD. When removing the 'altered mood and cognitions' subscale from the PTSD symptom checklist, the pattern of results was the same. After controlling for baseline ICD-11 PTSD symptoms, the three cognitive processes explained a significant $12 \%$ of variance in concurrent complex features. As in the original analyses, concurrent PTSD symptoms $(\beta=.35, \quad p=.001)$ and maladaptive appraisals $(\beta=.52, p<.001)$ were the sole processes uniquely associated with concurrent baseline complex features. In the original longitudinal model, the pattern of results were again the same. That is, after controlling for ICD-11 baseline PTSD symptoms, the three baseline cognitive processes continued to explain a significant $10 \%$ of variance in 12 -month complex features. Again, maladaptive appraisals, but not PTSD symptoms, was the sole unique predictor of 12-month complex features $(\beta=.41, p=.01)$.

Carer-reported symptoms. As a final, particularly stringent, sensitivity test, we explored whether results would be replicated when using carer-reported child symptoms. Here results differed slightly. All three baseline child-reported cognitive processes (appraisals, memory, coping) were significantly associated with baseline carer-reported PTSD symptoms $(r s=.27-.33, \quad p s<.01)$ and complex features $(r s=.25-.40, p s<.02)$. In a regression, while the three processes explained a unique $17 \%$ of variance in concurrent carer report PTSD symptoms $(p=.002)$, no individual cognitive process was identified as a unique driver (maladaptive appraisals: $\beta=.26$, $p=.07$; coping: $\beta=.07, p=.65$; memory: $\beta=.16$, $p=.25)$. For concurrent complex features, the three cognitive processes explained a unique $19 \%$ of variance $(p=.001)$, with maladaptive appraisals identified as the sole unique driver $(\beta=.41, p=.005$; coping: $\beta=.02, p=.87$; memory: $\beta=.05, p=.74)$. When controlling for carer-reported PTSD symptoms, the child-reported cognitive processes no longer predicted carer-reported complex features $(p=.66)$.

The only significant association between initial child-reported cognitions and 12-month carer-reported PTSD symptoms and complex features was that initial maladaptive appraisals were significantly associated with 12-month complex features $(r=.27$, $p=.02)$. In the regression model, after controlling for sex, baseline maladaptive appraisals remained a unique driver of 12-month carer-reported complex feature severity $(\beta=.29, p=.02)$. This was no longer the case after controlling for baseline carer-reported symptom severity $(p=.13)$.

\section{Discussion}

We explored the relevance of existing cognitive models of PTSD in relation to both 'standard' PTSD symptoms and complex features, in a sample of young people in out-of-home care. All participants had been exposed to trauma or maltreatment. On average they had entered care at approximately 8 years of age, meaning in many cases, these experiences had been prolonged. Based on the young person's self-report, we found both high and chronic levels of PTSD symptoms, which were strongly correlated with complex feature symptoms. There was good evidence for the relevance of existing cognitive models of PTSD, with the same processes associated with PTSD symptoms and complex features, and maladaptive appraisals identified as particularly important driver of symptoms.

Cognitive models of PTSD have been well tested in samples of adults (e.g. following sexual assault: Dunmore et al., 2001) and also with children 
exposed to single-incident traumas (e.g. Bryant et al., 2007; Meiser-Stedman et al., 2019). Here, we found good evidence that these same models are also applicable in more complex child trauma cases. Selfreported higher maladaptive appraisals, disorganised and sensory-heavy memories, and maladaptive cognitive coping strategies (i.e. avoidance, rumination) were all robustly associated with both young person and carer-reported concurrent PTSD symptoms and complex features, and child-reported longer-term (12-month) outcomes. These processes were more important in relation to symptom severity, than the young person's maltreatment severity and age they entered care (as a proxy of duration of exposure). These findings are consistent with the broader child trauma field that peri- and posttrauma variables tend to be more robust predictors of PTSD symptoms, than objective trauma severity and pretrauma variables (Trickey et al., 2012). When appraisals, coping and memory factors were examined simultaneously as predictors, maladaptive appraisals and to a lesser consistent extent, maladaptive coping, were identified as the key drivers of both standard child-reported PTSD symptoms and complex features. Findings add to the growing evidence of the central role of maladaptive appraisals, in particular, as a key driver of young people's trauma-related mental health outcomes (de Haan et al., 2019; Mitchell et al., 2017).

That existing cognitive models of PTSD (e.g. Ehlers $\&$ Clark, 2000) were found to be relevant for young people in care and that the same processes drove both PTSD symptoms and complex features have important clinical implications. First, our findings largely dispel the belief that existing cognitive models are not relevant to young people exposed to complex trauma. Results show that maladaptive appraisals, avoidant coping and trauma memory qualities are important treatment targets for young people in care, where they present with elevated PTSD symptoms. Second, our results suggest that the same models are also appropriate for complex PTSD and that the same treatment targets are therefore potentially appropriate. Efficacious treatment approaches already exist for targeting the core psychological processes that maintain PTSD symptoms and complex features, with traumafocused CBT (TF-CBT) the NICE-recommended firstline approach, even in cases of more complex trauma (see Cohen et al., 2012; Leenarts, Diehle, Doreleijers, Jansma, \& Lindauer, 2013; NICE, 2018). Recent work has demonstrated that this treatment approach reduces PTSD symptoms at the same rate for young people with PTSD and complex PTSD, although those with complex PTSD still had significantly higher PTSD symptoms at the end of treatment relative to young people who met criteria for PTSD only (Sachser, Keller et al., 2017). Our findings suggest that a stronger focus (e.g. more treatment sessions) on maladaptive appraisals may be a useful avenue for further improving outcomes for these more complex cases.
There are significant practical barriers to young people accessing evidence-based trauma-focused intervention (e.g. poor availability of services due to poor funding). However, there is also some evidence that when clinicians are trained to provide TF-CBT to maltreated young people, they are more likely to exclude components that cover cognitive restructuring and trauma narratives, in favour of components such as relaxation training (Allen \& Johnson, 2012). The current study has shown that the elements of TF-CBT that address maladaptive appraisals are likely to be central to addressing PTSD in this group. Further work is needed on understanding and overcoming potential implementation barriers both in terms of TF-CBT more broadly, and specifically the cognitive components. The fact that elevated PTSD and complex feature symptoms were relatively common in our sample, from the perspectives of both young people and carers, demonstrates the importance of ensuring this group are being adequately screened and assessed, to support decision-making around referrals and treatments of trauma-related mental health difficulties (Lewis et al., 2019).

This work has a number of strengths, including the longitudinal design and the focus on a complex and under-researched group. Nevertheless, results should be interpreted in light of limitations. First, young people reported on both the mechanisms and symptoms, which introduces single informant bias. When using carer report of symptoms results were less robust, with little evidence of longitudinal effects. At the same time, child self-report is recommended when reporting on internal states (MeiserStedman et al., 2007), while there is also the added complexity in this sample, that in some cases the carer may not have known the young person for very long. Indeed, there was poor agreement between children and carers in terms of both symptoms and maltreatment exposure. In contrast to the broader child trauma field (e.g. Meiser-Stedman et al., 2007), we found caregivers reported higher symptoms than young people. Findings demonstrate the importance of gathering information from multiple sources, which should include the young person (Kaufman et al., 1994). It would be useful for future research to explore what carer versus young person report differentially predict (e.g. in terms of functional outcomes). Second, while dropout was not associated with the young person's baseline PTSD symptoms or complex feature severity, we cannot rule out that there may have been some selective dropout for reasons that we failed to capture (e.g. other mental health difficulties), particularly given those who did not complete follow-up tended to be slightly older. Third, this study did not use diagnostic interviews to assess PTSD. Thus, rates of probable PTSD should be treated with caution. Similarly, while not the focus of this research, it is worth noting that it is highly likely that young people with elevated PTSD, particularly alongside complex features, may have 
also been experiencing other comorbidities. Comorbidities are commonplace alongside PTSD and further understanding the diagnostic and treatment implications of comorbidities remains an important area of research. Relatedly, complex PTSD is a new diagnosis and further research is needed to explore whether this should remain a unique diagnosis or be considered a marker for more severe PTSD, where comorbidities and significant functional impairment may be more common. That complex PTSD is a new diagnosis also means the complex feature questionnaire for assessing its unique symptoms has not yet been fully validated. That we showed it correlates with PTSD symptoms and trauma-specific cognitions, and has good internal consistency, demonstrates promising preliminary psychometric evidence for this scale.

To conclude, we found good evidence that existing cognitive models of PTSD are applicable to both PTSD and complex features, in a sample of young people who have experienced maltreatment and live in out-ofhome care. These young people are some of the most vulnerable UK youth, and their poor mental health and well-being outcomes have been long-documented. Our results show that, where these young people are experiencing elevated PTSD symptoms, regardless of the presence or absence of complex features, treatment targets should particularly include maladaptive appraisals, while trauma memory quality and coping strategies are also important. These are the treatment targets in the established trauma-focused CBT (NICE, 2018), with our mechanism results supporting this treatment as the first-line approach for PTSD, including for young people in care.

\section{Supporting information}

Additional supporting information may be found online in the Supporting Information section at the end of the article:

Figure S1. Child complex PTSD checklist.

Table S1. Bivariate and point biserial correlations between descriptives, maltreatment severity, PTSD and complex features.

Table S2. Bivariate correlations between baseline cognitive processes and child-reported PTSD symptoms (PTSS) and complex features severity at each assessment point.

Table S3. Longitudinal pooled regression results for cognitive processes predicting PTSS and complex features, following multiple-imputation.

\section{Acknowledgements}

This project was funded by an ESRC Future Leader grant (ES/NO1782X/1) and University of Bath Prize Fellowship awarded to R.M.H. The authors wish to sincerely thank all of the staff at the participating social care sites for their enthusiasm and commitment to this work, and to improving mental health outcomes for these young people. We also particularly wish to thank the young people and carers who volunteered their time for this project.

\section{Correspondence}

Rachel M. Hiller, Department of Psychology, University of Bath, Claverton Down, Bath, BA2 7AY, UK; Email: R.Hiller@bath.ac.uk

\section{Key points}

- Children in foster care have well-documented poor mental health outcomes, yet there is little quantitative evidence of what processes may maintain these difficulties. There remains ongoing debate about the relevance of existing models of psychopathology for these young people, especially for cognitive and behavioural models.

- Given many have been exposed to trauma or maltreatment, one potential psychological outcome is PTSD, as well as the newly introduced complex PTSD. We sought to explore whether existing cognitive models of PTSD would be applicable to this group, both in relation to 'standard' PTSD symptoms and the additional complex features.

- Our longitudinal study found good evidence that cognitive models of PTSD are applicable to both 'standard' PTSD symptoms and the additional features of complex PTSD. Maladaptive appraisals, memory qualities and coping (e.g. avoidance) were associated with concurrent and longer-term (12-month) PTSD and complex features, with maladaptive appraisals particularly important.

- That these mechanisms were found to maintain PTSD symptoms and complex features, suggest they would be useful treatment targets for young people in care with elevated PTSD, with or without complex features.

- This study also showed poor agreement between young people and carers about PTSD and complex feature symptoms, highlighting the importance of services collecting young person self-report on their own mental health, rather than solely relying on carer report. 


\section{References}

Allen, B., \& Johnson, J.C. (2012). Utilization and implementation of trauma-focused cognitive-behavioral therapy for the treatment of maltreated children. Child Maltreatment, $17,80-85$.

American Psychiatric Association. (2013). Diagnostic and statistical manual of mental disorders (5th edn). Arlington, VA: Author.

Brewin, C.R. (2016). Coherence disorganization and fragmentation in traumatic memory reconsidered: A response to Rubin et al (2016). Journal of Abnormal Psychology, 125, 1011-1017.

Brewin, C.R., Dalgleish, T., \& Joseph, S. (1996). A dual representation theory of posttraumatic stress disorder. Psychological Review, 103, 670.

Brewin, C.R., Cloitre, M., Hyland, P., Shevlin, M., Maercker, A., Bryant, R.A., .. \& \& Somasundaram, D. (2017). A review of current evidence regarding the ICD-11 proposals for diagnosing PTSD and Complex PTSD. Clinical Psychology Review, 58, 1-15.

Bryant, R.A., Salmon, K., Sinclair, E., \& Davidson, P. (2007). A prospective study of appraisals in childhood posttraumatic stress disorder. Behaviour Research and Therapy, 45, 2502-2507.

Cloitre, M., Garvert, D.W., Brewin, C.R., Bryant, R.A., \& Maercker, A. (2013). Evidence for proposed ICD-11 PTSD and Complex PTSD: A latent profile analysis. European Journal of Psychotraumatology, 4, 20706.

Cohen, J.A., Mannarino, A.P., Berliner, L., \& Deblinger, E. (2000). Trauma-focused cognitive behavioral therapy for children and adolescents: An empirical update. Journal of Interpersonal Violence, 15, 1202-1223.

Cohen, J.A., Mannarino, A.P., Kliethermes, M., \& Murray, L.A. (2012). Trauma-focused CBT for youth with complex trauma. Child Abuse \& Neglect, 36, 528-541.

Dalgleish, T., Meiser-Stedman, R., \& Smith, P. (2005). Cognitive aspects of posttraumatic stress reactions and their treatment in children and adolescents: An empirical review and some recommendations. Behavioural and Cognitive Psychotherapy, 33, 459-486.

de Haan, A., Tutus, D., Goldbeck, L., Rosner, R., \& Landolt, M.A. (2019). Do dysfunctional posttraumatic cognitions play a mediating role in trauma adjustment? Findings from interpersonal and accidental trauma samples of children and adolescents. European Journal of Psychotraumatology, 10,1596508 .

DeJong, M. (2010). Some reflections on the use of psychiatric diagnosis in the looked after or "in care" child population. Clinical Child Psychology and Psychiatry, 15, 589-599.

Department for Education. (2018). Statistics on children under local authority care. London: Author.

Dunmore, E., Clark, D.M., \& Ehlers, A. (2001). A prospective investigation of the role of cognitive factors in persistent posttraumatic stress disorder (PTSD) after physical or sexual assault. Behaviour Research and Therapy, 39, 1063-1084.

Ehlers, A., \& Clark, D.M. (2000). A cognitive model of posttraumatic stress disorder. Behaviour Research and Therapy, 38, 319-345.

Foa, E.B., Steketee, G., \& Rothbaum, B.O. (1989). Behavioral/ cognitive conceptualizations of posttraumatic stress disorder. Behavior Therapy, 20, 155-176.

Ford, T., Vostanis, P., Meltzer, H., \& Goodman, R. (2007). Psychiatric disorder among British children looked after by local authorities: comparison with children living in private households. The British Journal of Psychiatry, 190, 319-325.

Gonzales, N.A., Tein, J.Y., Sandler, I.N., \& Friedman, R.J. (2001). On the limits of coping: Interaction between stress and coping for inner-city adolescents. Journal of Adolescent Research, 16, 372-395.

Grant, K.E., O’Koon, J.H., Davis, T.H., Roache, N.A., Poindexter, L.M., Armstrong, M.L., ... \& McIntosh, J.M. (2000).
Protective factors affecting low-income urban African American youth exposed to stress. The Journal of Early Adolescence, 20, 388-417.

Hiller, R.M., Creswell, C., Meiser-Stedman, R., Lobo, S., Cowdrey, F., Lyttle, M.D., ... \& Halligan, S.L. (2018). A longitudinal examination of the relationship between trauma-related cognitive factors and internalising and externalising psychopathology in physically injured children. Journal of Abnormal Child Psychology, 47, 683-693.

Hiller, R.M., \& St Clair, M.C. (2018). The emotional and behavioural symptom trajectories of children in long-term out-of-home care in an English local authority. Child Abuse \& Neglect, 81, 106-117.

Karatzias, T., Cloitre, M., Maercker, A., Kazlauskas, E., Shevlin, M., Hyland, P., ... \& Brewin, C.R. (2017). PTSD and Complex PTSD: ICD-11 updates on concept and measurement in the UK, USA, Germany and Lithuania. European Journal of Psychotraumatology, 8, 1418103.

Kaufman, J., Jones, B., Stieglitz, E., Vitulano, L., \& Mannarino, A.P. (1994). The use of multiple informants to assess children's maltreatment experiences. Journal of Family Violence, 9, 227-248.

Keyes, K.M., Eaton, N.R., Krueger, R.F., McLaughlin, K.A., Wall, M.M., Grant, B.F., \& Hasin, D.S. (2012). Childhood maltreatment and the structure of common psychiatric disorders. The British Journal of Psychiatry, 200, 107-115.

Leenarts, L.E., Diehle, J., Doreleijers, T.A., Jansma, E.P., \& Lindauer, R.J. (2013). Evidence-based treatments for children with trauma-related psychopathology as a result of childhood maltreatment: a systematic review. European Child \& Adolescent Psychiatry, 22, 269-283.

Lewis, S.J., Arseneault, L., Caspi, A., Fisher, H.L., Matthews, T., Moffitt, T.E., .. \& \& Danese, A. (2019). The epidemiology of trauma and post-traumatic stress disorder in a representative cohort of young people in England and Wales. The Lancet Psychiatry, 6, 247-256.

McKinnon, A., Brewer, N., Meiser-Stedman, R., \& Nixon, R.D.V. (2017). Trauma memory characteristics and the development of acute stress disorder and post-traumatic stress disorder in youth. Journal of Behavior Therapy and Experimental Psychiatry, 54, 112-119.

Meiser-Stedman, R., McKinnon, A., Dixon, C., Boyle, A., Smith, P., \& Dalgleish, T. (2019). A core role for cognitive processes in the acute onset and maintenance of posttraumatic stress in children and adolescents. Journal of Child Psychology and Psychiatry, 60, 875-884.

Meiser-Stedman, R., Smith, P., Glucksman, E., Yule, W., \& Dalgleish, T. (2007). Parent and child agreement for acute stress disorder, post-traumatic stress disorder and other psychopathology in a prospective study of children and adolescents exposed to single-event trauma. Journal of Abnormal Child Psychology, 35, 191-201.

Meiser-Stedman, R., Smith, P., Bryant, R., Salmon, K., Yule, W., Dalgleish, T., \& Nixon, R.D. (2009). Development and validation of the child post-traumatic cognitions inventory (CPTCI). Journal of Child Psychology and Psychiatry, 50, 432-440.

Mitchell, R., Brennan, K., Curran, D., Hanna, D., \& Dyer, K.F. (2017). A meta-analysis of the association between appraisals of trauma and posttraumatic stress in children and adolescents. Journal of Traumatic Stress, 30, 88-93.

National Institute of Health and Care Excellence (NICE) (2018). Post-traumatic stress disorder (NICE Guideline 116). Available from: https://www.nice.org.uk/guidance/ng116 [last accessed February 2019].

Oswald, S.H., Heil, K., \& Goldbeck, L. (2009). History of maltreatment and mental health problems in foster children: A review of the literature. Journal of Pediatric Psychology, 35, 462-472.

Pietrzak, R.H., Harpaz-Rotem, I., \& Southwick, S.M. (2011). Cognitive-behavioral coping strategies associated with 
combat-related PTSD in treatment-seeking OEF-OIF veterans. Psychiatry Research, 189, 251-258.

Rock, S., Michelson, D., Thomson, S., \& Day, C. (2013). Understanding foster placement instability for looked after children: A systematic review and narrative synthesis of quantitative and qualitative evidence. British Journal of Social Work, 45, 177-203.

Sachser, C., Keller, F., \& Goldbeck, L. (2017). Complex PTSD as proposed for ICD-11: Validation of a new disorder in children and adolescents and their response to TraumaFocused Cognitive Behavioral Therapy. Journal of Child Psychology and Psychiatry, 58, 160-168.

Sachser, C., Berliner, L., Holt, T., Jensen, T.K., Jungbluth, N., Risch, E., .. \& Goldbeck, L. (2017). International development and psychometric properties of the Child and Adolescent Trauma Screen (CATS). Journal of Affective Disorders, 210, 189-195.

Smith, D.K., Johnson, A.B., Pears, K.C., Fisher, P.A., \& DeGarmo, D.S. (2007). Child maltreatment and foster care: Unpacking the effects of prenatal and postnatal parental substance use. Child Maltreatment, 12, 150-160.
Stallard, P., \& Smith, E. (2007). Appraisals and cognitive coping styles associated with chronic post-traumatic symptoms in child road traffic accident survivors. Journal of Child Psychology and Psychiatry, 48, 194-201.

Trickey, D., Siddaway, A.P., Meiser-Stedman, R., Serpell, L., \& Field, A.P. (2012). A meta-analysis of risk factors for posttraumatic stress disorder in children and adolescents. Clinical Psychology Review, 32, 122-138.

Van der Kolk, B.A. (2017). Developmental Trauma Disorder: Toward a rational diagnosis for children with complex trauma histories. Psychiatric Annals, 35, 401-408.

Wiseman, H., Hamilton-Giachritsis, C., \& Hiller, R.M. (2019). The relevance of cognitive behavioral models of post-traumatic stress following child maltreatment: a systematic review. Trauma, Violence, \& Abuse, In Press.

World Health Organisation. (2018). International statistical classification of diseases and related health problems (11th Revision). Available from: https://icd.who.int/browse11/ $1-\mathrm{m} /$ en [last accessed June 2019].

Accepted for publication: 11 February 2020 